\title{
Genetic analysis in a patient with nine primary malignant neoplasms: A rare case of Li-Fraumeni syndrome
}

\author{
XIAOYUAN LI ${ }^{1 *}$, JUAN KANG ${ }^{2 *}$, QI PAN ${ }^{2 *}$, WERONIKA SIKORA-WOHLFELD ${ }^{3}$, \\ DACHUN ZHAO ${ }^{4}$, CHANGTING MENG $^{1}$, CHUNMEI BAI $^{1}$, ANIL PATWARDHAN $^{5}$, \\ RICHARD CHEN ${ }^{5}$, HONG REN ${ }^{2}$, ATUL J. BUTTE ${ }^{3}$ and KEYUE DING ${ }^{2}$
}

\begin{abstract}
${ }^{1}$ Department of Medical Oncology, Peking Union Medical College Hospital, Peking Union Medical College and Chinese Academy of Medical Sciences, Beijing; ${ }^{2}$ Institute for Viral Hepatitis, Key Laboratory of Molecular Biology for Infectious Diseases, Ministry of Education of China; Department of Infectious Diseases, The Second Affiliated Hospital of Chongqing Medical University, Chongqing, P.R. China; ${ }^{3}$ Division of Systems Medicine, Department of Pediatrics, Stanford University, CA, USA; ${ }^{4}$ Department of Pathology, Peking Union Medical College Hospital, Peking Union Medical College and Chinese Academy of Medical Sciences, Beijing, P.R. China; ${ }^{5}$ Personalis, Inc., Menlo Park, CA, USA
\end{abstract}

Received October 14, 2015; Accepted November 29, 2015

DOI: $10.3892 / o r .2015 .4501$

\begin{abstract}
To identify rare mutations and retrospectively estimate the cancer risk of a 45 -year old female patient diagnosed with Li-Fraumeni syndrome (LFS), who developed nine primary malignant neoplasms in a period of 38 years, we conducted next-generation sequencing in this patient. Whole-genome and whole-exome sequencing were performed in DNA of whole blood obtained a year prior to the diagnosis of acute myeloid leukemia (AML) and at the time of diagnosis of AML, respectively. We analyzed rare mutations in cancer susceptibility genes using a candidate strategy and estimated cancer risk using the Risk-O-Gram algorithm. We found rare mutations in cancer susceptibility genes associated with an increased hereditary cancer risk in the patient. Notably, the number of mutated genes in p53 signaling pathway was significantly higher than expected $(\mathrm{P}=0.02)$. However, the phenotype of multiple malignant neoplasms of the studied patient was unlikely to be caused by accumulation of common cancer risk alleles. In conclusion, we established the mutation profile in a rare case of Li-Fraumeni syndrome, illustrating that the rare mutations rather than the cumulative of common risk alleles leading to an increased cancer risk in the patient.
\end{abstract}

Correspondence to: Dr Keyue Ding, Institute for Viral Hepatitis, Key Laboratory of Molecular Biology for Infectious Diseases, Ministry of Education of China; Department of Infectious Diseases, The Second Affiliated Hospital of Chongqing Medical University, 76 Road Linjiang, Yuzhong Chongqing, 400010, P.R. China

E-mail: ding.keyue@cqmu.edu.cn

${ }^{*}$ Contributed equally

Key words: Li-Fraumeni syndrome, next-generation sequencing, mutation, susceptibility, cancer risk

\section{Introduction}

Li-Fraumeni syndrome (LFS) (OMIM \#151623), a heterogeneous inherited autosomal dominant disorder, manifested by the occurrence of soft tissue sarcomas, early-onset breast cancer and other neoplasms in children and young adults (1). There is an exceptionally high risk of developing multiple primary cancers in members of families with LFS (2). For example, among 200 LFS family members diagnosed with cancer, $15 \%$ developed a second, $4 \%$ a third and $2 \%$ developed a fourth cancer (2). The genetic defect responsible for LFS lies in the germline mutations in the tumor suppressor gene TP53 (3). Germline mutations in CHEK2 may predispose to a few LFS-like families (4). A third LFS locus (LFS3) has been mapped to chromosome 1q23 but no specific gene has yet been identified (5).

A 45-year old female patient, who had a family history of breast cancer, developed neuroblastoma, rhabdomyosarcoma, breast, lung, thyroid, and gastric cancer, osteosarcoma, as well as acute myeloid leukemia (AML) in a period of 38 years. This is a rare case of LFS with nine metachronous tumors. The family presented with features suggesting LFS (the Chompret criteria). It is unknown how the patient developed nine malignant tumors, but survived 45 years.

In the present study, we performed whole-genome sequencing from blood specimens obtained a year prior to the diagnosis of AML, as well as whole-exome sequencing from blood specimens at the time of diagnosis of AML in the subject. We aimed to obtain a better understanding of: i) how rare mutations in the genome contribute to the development of the cancer phenotypes; ii) how the cancer risk can be retrospectively estimated by common variants; and iii) why the patient survival was longer than expected in LFS patients.

\section{Materials and methods}

DNA samples. DNA samples from the patient's whole blood were banked for next-generation sequencing under a protocol 
approved by the institutional review board (IRB) at the Peking Union Medical College Hospital (PUMCH). One sample was obtained a year prior to the diagnosis of acute myeloid leukemia (AML)-M $\mathrm{M}_{2}$ (pre-AML), and another sample was obtained at the time of the diagnosis of AML-M $\mathrm{M}_{2}$. The patient provided written informed consent.

DNA extraction, library construction and next-generation sequencing. Genomic DNA was isolated from two samples of whole blood (pre-AML-DNA and AML-DNA). We performed whole-genome sequencing (WGS) and whole-exome sequencing (WES) for pre-AML-DNA and AML-DNA, respectively. All sequencing was carried out at the Beijing Genomic Institute at Shenzhen (BGI-Shenzhen, Shenzhen, China). A detailed description of library preparation and sequencing is available upon request.

Sanger sequencing for TP53. We used the International Agency for Research on Cancer (IARC) protocol (p53.iarc.fr/ Download/TP53_DirectSequencing_IARC.pdf) for detection and validation of mutations in exons (2-11) of TP53 by Sanger sequencing.

Identification of single nucleotide variants (SNVs), structural variants (SVs) and somatic mutations. A computational pipeline was used for variant discovery. The pipeline integrates software tools widely used for analyzing next-generation sequencing data. For detecting structural variants, we used BreakDancer (6) to predict a wide variety of structural variants including large-scale indels, inversions and intra-/inter-chromosomal translocations. We used Control-FREEC (7) for assessing copy number variants. The program MuTect (8) was used to identify somatic mutations leading to leukemic transformation by comparing pre-AML DNA and AML DNA.

Analysis of rare mutations. In the case of only a single dominantly affected individual available, we designed a pipeline to prioritize the filtered mutations based on a candidate strategy (9). Analysis-ready variants were annotated using SeattleSeq annotation (http://snp.gs.washington.edu/ SeattleSeqAnnotation138/). We focused on three areas: i) variants associated with genes for hereditary cancer predisposition syndrome, i.e., syndromes of inherited cancer predisposition in clinical oncology syndrome (10); ii) variants in DNA repair genes (11); and iii) mutations in TP53 and genes in the p53 signaling pathway (KEGG, www.genome.jp/kegg-bin/ show_pathway?map04115). We performed database queries, biophysical prediction and analyses of non-coding regions to screen for known and novel mutations. For rare novel mutations, we prioritized variants for stop, frame-shift and splice site mutations. Polyphen (12) was used to predict the functional effect of missense mutations.

Genetic risk prediction based on common variants. To retrospectively estimate the cancer risk, we performed genetic risk prediction using the VARiants Informing MEDicine (VARIMED) database, a manually curated database of human SNP-disease associations (13). The current VARIMED database contained 466,890 unique SNPs associated with 6,691 diseases and related traits extracted from 17,088 publica- tions. We extracted all variants from VARIMED that were associated with phenotypes containing terms of 'cancer', 'lymphoma', 'leukemia', 'melanoma', 'glioma', 'carcinoma', 'neuroblastoma', 'myeloma', 'sarcoma' and 'glioblastoma'. We only used SNP-disease associations that were observed in at least two genome-wide association studies at the significance level $\mathrm{P}<10^{-6}$ with total number of at least 2,000 participants.

We estimated the pre-test probability of developing different tumors in Chinese women using the incidence (agestandardized incidence rate) reported in 2012 GLOBOCAN (globocan.iarc.fr/Pages/fact_sheets_population.aspx). For all SNPs associated with a given cancer, we emitted all genotypes (variant calls and reference-reference calls) from the WES data. We determined likelihood ratio (LR) for each SNP-disease association based on VARIMED, correcting for the effects of linkage disequilibrium among multiple loci (14). Finally, we combined the pre-test probabilities and LRs to calculate the post-test probabilities (14). We performed risk prediction analysis for the patient, as well as for all Chinese individuals from the 1000 Genomes Project phase 1 as a background (CHB and CHS populations, $n=197$ ) (15).

We assigned a unique risk allele for each cancer-related SNPs (if an SNP was associated with multiple cancers with different risk alleles, we chose the risk allele linked to the majority of the cancers). The total number of risk alleles was calculated in the patient's exome and in each of the 197 genomes of Chinese populations from the 1000 Genomes data.

\section{Results}

Patient. The patient was a 45-year old female Han Chinese, who presented with nine primary malignant neoplasms (Fig. 1A and B) and a family history of breast cancer (Fig. 1C). At the age of 7 , the patient was subsequently operated for upper lip neuroblastoma (received postoperatively local radiotherapy) and rhabdomyosarcoma. Twenty-one years later, she was treated for cystosarcoma phyllodes of the right breast by radical mastectomy and postoperative radiotherapy. At the age of 29, after a lung mass was found, the left-upper lobe resection and mediastinal lymphadenectomy revealed bronchioalveolar cell carcinoma with hilar lymphatic metastases (T3N1M0, IIIA). She received postoperatively adjuvant chemotherapy. Ten years later (at the age of 39), she found a left breast mass by self-examination and a modified radical mastectomy was performed. The pathology analysis showed a middle-differentiated infiltrating ductal carcinoma (T2N0M0, IIA). She was given adjuvant chemotherapy and endocrine therapy. Five months later, after pharynx discomfort, she was treated for thyroid nodule with total thyroidectomy for the left thyroid and near total thyroidectomy for the right thyroid. Pathology reports indicated nodular goiter and papillary carcinoma. At the age of 41 , she received gastric endoscopy due to black stool and found preventriculus ulcer. She was treated by radical gastrectomy and indicated gastric adenocarcinoma (T4N1M0, IIIA), and she received four cycles of chemotherapy (paclitaxel and cisplatin). Two months later, the bone scanning indicated 'abnormality in the maxilla' and surgical resection of the mass in the right maxilla showed osteosarcoma. Two years later (at the age of 43), the patient found a lump on the sternum and the bone scanning showed an abnormal radioac- 
A

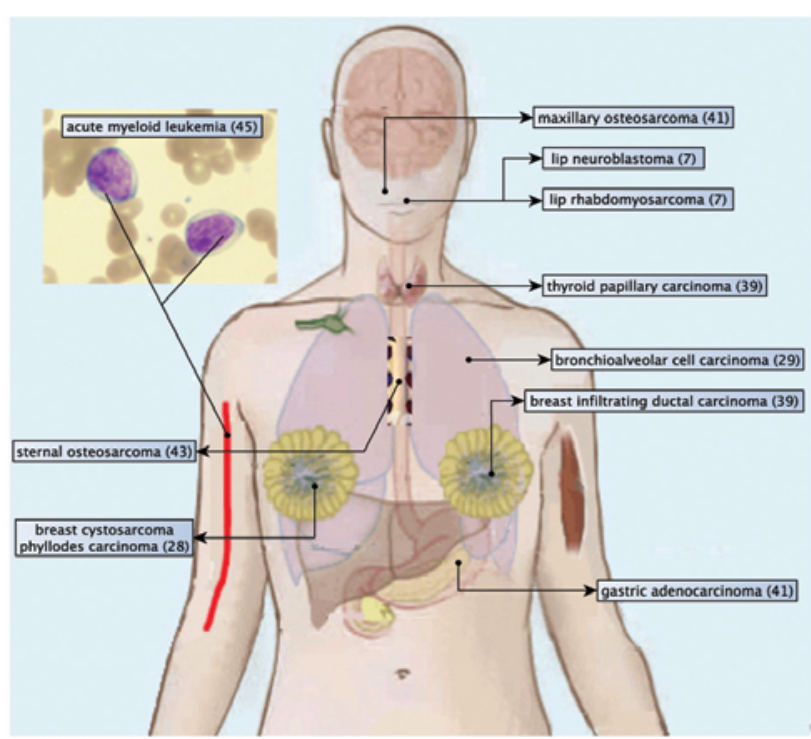

C

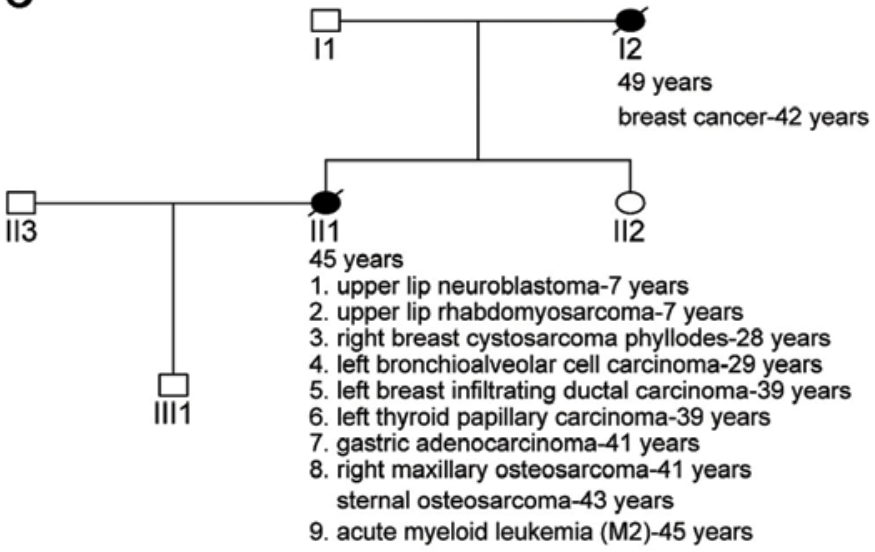

B

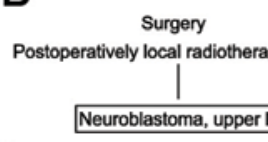

Year

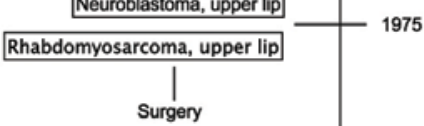

Surgery
Postoperative chemotherapy: mitomycin, cisplatin /carboplatin, etoposide, 3 cycles Mediastinal lymphadenectomy Left-upper lobe resection
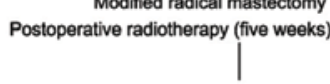

Cystosarcoma phyllodes,right breast

\section{Bronchioalveolar cell carcinoma, left lung}

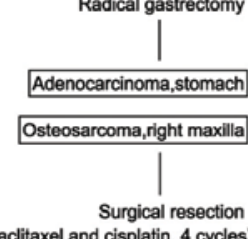

Postoperative chemotherapy (paclitaxel and cisplatin, 4 cycles)

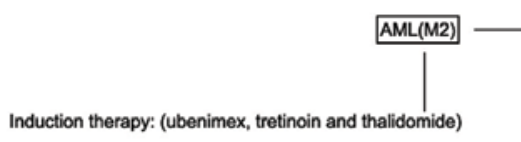

Endocrine therapy

Postoperative chemotherapy (CTF regimen, 6 cycles Modified radical mastectomy 2005

Infiltrating ductal carcinoma, left breast

Occult papillary carcinoma, thyroid

ear total thyroidectomy (right)

Total thyroidectomy (left)

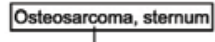

Week 10, 25, 29, 34: epirubicin+cisplatin

Week 5, 8: ifosfamide+cisplatin;

Week3,4: methotrexate;

Week1: pirarubicin;

Chemotherapy

Figure 1. (A) Localization of different tumors. The diagram of human body was taken and abridged from Fig. 1 in Vogelstein et al (42) with modifications. (B) The timelines of cancer development in different organs and treatments received by the patient. Boxes indicated the diagnosed cancers. (C) Pedigree structure of the affected family indicated a dominant mode of inheritance. The proband (II1) is a 45-year old female and was assessed in the present study. Solid oval (female) denoted affected individuals. Diagonal lines indicated deceased individual. Type of cancer and age of diagnosis or death are shown.

tive accumulation in the right sternoclavicular articulation. Sternal biopsy indicated spindle cell sarcoma (T1N0M0G3, IIA). We could not confirm that the sternal spindle cell sarcoma was a primary or a metastasis from the maxillary osteosarcoma. Without a chance for operation, the patient was administered chemotherapy and then local radiotherapy. At the age of 45 , analysis of her bone marrow revealed $30 \%$ myeloblasts and $13.5 \%$ promyelocytes and a complex karyotype involving monosomy 13,16 , and $\mathrm{X}$, trisomy 8,11 , $\operatorname{del}(5 \mathrm{q} 31), ? \operatorname{del}(17 \mathrm{p} 10), \mathrm{t}(1: ?)(\mathrm{q} 11: ?), \operatorname{ins}(1 \mathrm{p} 10), ? \operatorname{ins}(17 \mathrm{q} 10)$ and two marker chromosomes that could not be resolved by standard cytogenetic analysis. The patient developed respiratory infection and died five months later after the diagnosis of AML- $\mathrm{M}_{2}$. All clinical information, including photomicrographs in pathology, has been uploaded to the Phenomecentral database at https://phenomecentral.org/P0001643.

Thus, a total of at least nine primary malignant neoplasms developed within a 38 -year period. This rare LFS patient 


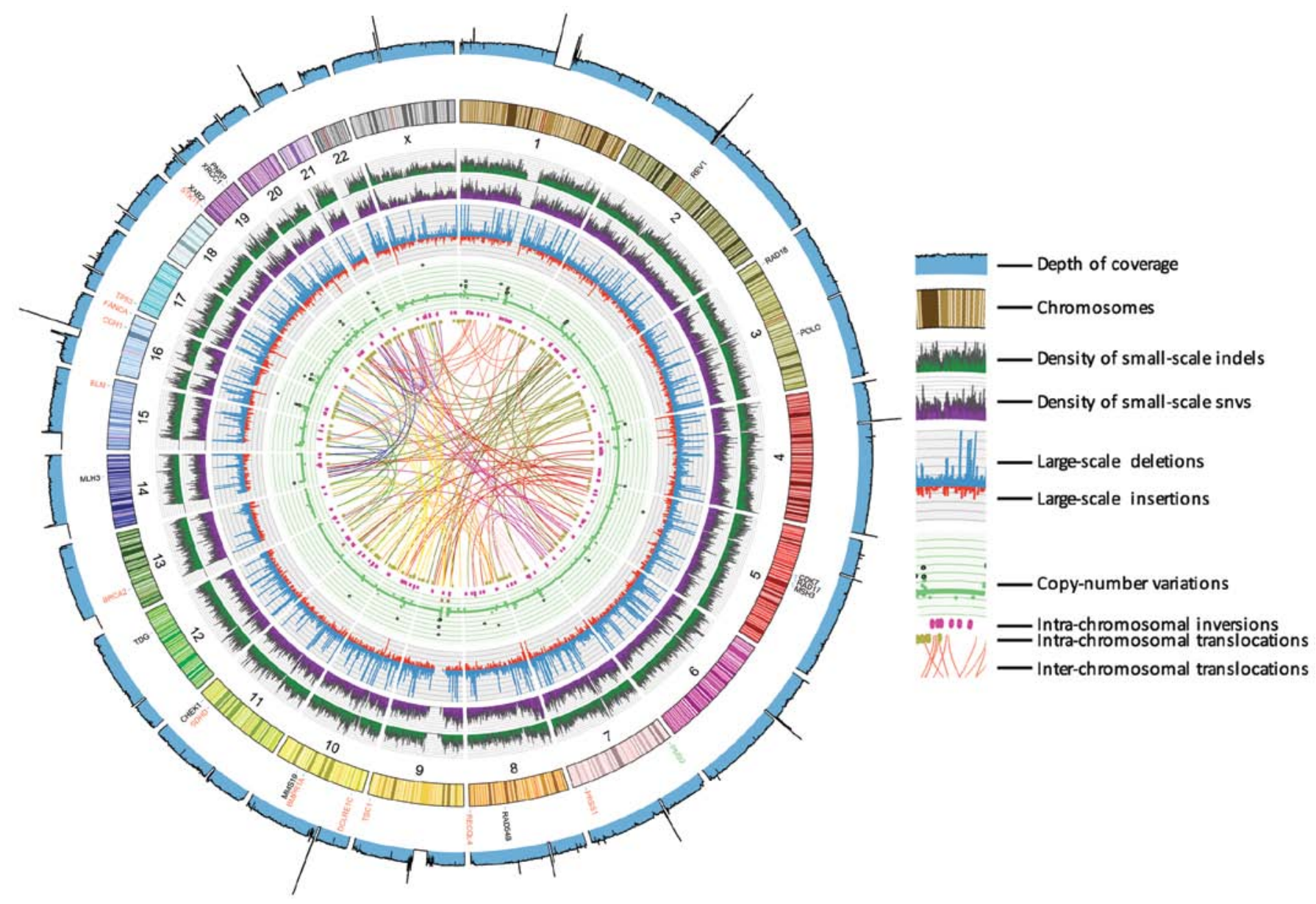

Figure 2. Circos plot summarizing the overall patterns of genetic variants identified by WGS. From inner to outer rings: inter-chromosomal translocations, the circles from inside going outside represent intra-chromosomal translocations (brown), inversions (red), copy-number variations (black stroke indicates copy number above 5, red stroke indicate copy number below 0 and green indicates 0 to 5), large-scale indels (the histogram represents insertion and deletion, the proportional length of indels is shown with orange-insertions facing inwards and blue-deletions facing outwards), the density of SNVs (purple) and small indels (green), chromosome ideogram (colorful bands), and the depth of coverage (blue). Genes listed in Table II were labeled according to their chromosomal locations.

provided a model to identify susceptibility rare mutations and estimate cancer risk based on common variants with the advent of next-generation sequencing. We performed wholegenome sequencing (WGS) from blood specimens obtained a year prior to the diagnosis of AML to create a reference sequence (germline) for this patient with a relatively lower coverage $(\sim \times 30)$ and used this reference sequence to investigate germline abnormity, including SNVs and aberrations. In order to estimate cancer-risk of this patient, we then performed whole-exome sequencing (WES) from blood specimens at the time of diagnosis of AML with a relatively higher coverage (105x). We can also detect somatic mutations underlying leukemic transformation in the patient by comparing with the reference sequence obtained a year prior to the diagnosis of AML.

Whole-genome sequencing (WGS). We performed four paired-end sequencing runs for pre-AML-DNA and produced 1,079,697,548 clean sequence reads (219.7 GB). In total, $96.88 \%$ of the reads were mapped to the hg 19 , resulting $99.14 \%$ total coverage of the reference genome. The distribution of read depth indicated that the average depth was 29.44 and $98.8 \%$ of genomic positions had read depth $\geq 4$.
We analyzed $2.84 \mathrm{Gbp}$ of the reference genome for SNVs and identified 3,565,277 filtered SNVs (autosomes and chromosome $\mathrm{X}$ ). The number was in the range of 3-4 million SNVs in an average person (16). Of these, $4.8 \%$ were not represented in the dbSNP database (build 137) and were considered to be novel. The numbers of homozygous and heterozygous SNVs were 1,477,141 and 2,084,985, respectively. In protein-coding regions, 10,223 missense and 99 nonsense mutations were identified (Table I).

The overall patterns of genome alterations, including density of SNVs, small indels, and structural variants of whole chromosomes, were seen in this patient (Fig. 2). The mode of density plots of SNVs and indels, in each interval of $0.25 \mathrm{MB}$, are similar. There were 171 inter-chromosomal and 263 intra-chromosomal translocations, indicating that intrachromosomal translocations outnumber inter-chromosomal translocations. We identified 3,778 large-scale insertions and 11,540 large-scale deletions, respectively. In total, 6,663 CNVs were identified, which varied from -2 to 30 and most of the CNVs were around 2. As expected, high value of CNVs commonly involves high sequence depth. On the centromere and/or chromosomes-end regions, the depth of coverage is low or lacking. 
Table I. Number of SNVs identified by whole genome sequencing and whole exome sequencing.

\begin{tabular}{lcc}
\hline & \multicolumn{2}{c}{ Number of SNVs } \\
\cline { 2 - 3 } Class & $\begin{array}{c}\text { Whole-genome } \\
\text { sequencing }\end{array}$ & $\begin{array}{c}\text { Whole-exome } \\
\text { Sequencing }\end{array}$ \\
\hline Missense & 10,223 & 9,826 \\
Nonsense & 99 & 87 \\
SNPs in splice sites & 65 & 52 \\
Synonymous & 11,111 & 10,972 \\
SNPs in coding (not mod 3) & 27 & 28 \\
SNPs in a UTR & 28,659 & 10,126 \\
SNPs near a gene & 72,332 & 11,910 \\
Intronic & $1,256,089$ & 200,160 \\
Intergenic SNPs & $2,184,672$ & 208,248 \\
SNPs in microRNAs & 123 & 105 \\
SNPs in dbSNP & $3,480,654$ & 446,277 \\
SNPs not in dbSNP & 82,623 & 5,132 \\
Total & $3,565,277$ & 451,409 \\
\hline
\end{tabular}

\section{Analyses of rare mutations}

Mutations associated with hereditary cancer predisposition syndromes. We first searched for evidence of known/novel rare missense mutations ( $<5 \%$ of the minor allele frequency in Asians from HapMap) that would predispose the patient to hereditary cancer predisposition syndromes (10), including LFS cancers. We identified at least 20 known and 2 novel rare missense mutations within 13 genes lead to hereditary cancer predisposition syndromes, which may be associated with increased risk of cancer in the patient (Table II). High interest genes include: i) a mutation (rs28934576, R273H) in TP53, which was previously identified in LFS patients with softtissue sarcoma and gastric carcinoma $(17,18)$; ii) a mutation (rs144848, N372H) in BRCA2, associated with an increased risk of breast cancer (19); and iii) a probably-damaging missense mutation (rs2276331, L630V) in CDH1, where a heterozygous insertion in $\mathrm{CDHl}$ was found in the germline of a woman who developed lobular breast cancer (20) and a mutation in $C D H I$ increased risk of developing gastric cancer and other cancers associated with human diffuse gastric cancer (HDGC) (21). Additionally, several rare missense mutations were identified in the RecQ helicase family, i.e., RECQL4 (RecQ proteinlike 4), and BLM (Bloom syndrome, RecQ helicase-like). DNA helicase deficiencies has been found to be associated with chromosome instability and cancer predisposition (22). Mutations in RECQL4 and BLM have been shown to cause hereditary disorders, i.e., Rothmund-Thomson Syndrome (23), and Bloom Syndrome (24), respectively. Recently, WES identified rare deleterious mutations in $B L M$ as potential breast cancer susceptibility alleles (25). We also noted several rare missense mutations in genes associated hereditary gastrointestinal malignancies, e.g., Juvenile polyposis (BMPR1A), Turcot syndrome (PMS2) and Peutz-Jeghers syndrome (STK11). However, function effects of these missense mutations needed to be further studied.
Mutations in DNA repair genes. Defects of DNA repair are a common cause of inherited cancer susceptibility. DNA mismatch repair (MMR) is a system for recognizing and repairing mismatch (e.g., erroneous indels and mismatched bases) during DNA replication and recombination, as well as repairing some forms of DNA damage (26). We investigated whether the patient had mutations in DNA repair genes (Table II). We identified two rare missense mutations in PMS2, as well as one each in MSH3 and MLH3. The rs1805321 (P470S) in PMS2 was previously found in three out of seven patients with colorectal cancer without family history (27). We additionally found a common variant in the $M L H 1$ promoter (rs1800734, A/G), which was previously found to be heterozygous in a patient with metachronous carcinomas that had microsatellite instability and lacked $M L H 1$ expression (28).

Variants in TP53 and genes in the p53-signaling pathway. As the signaling cascades are genetically dysregulated tumor dependencies, we characterized variants in the p53-signaling pathway. Of 68 genes in the p53-signaling pathway, 19 missense mutations in 14 genes were identified, which was significantly higher than expected (Fisher's exact test, $\mathrm{P}=0.02$ ) (Fig 3). The mutated genes were clustered in: i) the mediators of p53 signaling pathway (e.g., ATM and CHEK1); ii) TP53; and iii) transducers leading to the outcomes of 'apoptosis' and 'inhibition of angiogenesis and metastasis'. Of note, two missense mutations (rs1042522 and rs28934576) in TP53 were identified and confirmed by Sanger sequencing. The rs 28934576 (codon 272, His293Arg) was identified previously to be present in a case of LFS with gastric cancer (18). We did not note other missense mutations in TP53.

Whole-exome sequencing (WES). WES was performed for AML-DNA with a relatively higher depth of coverage (105x); resulting in an output of $30 \mathrm{~GB}$ of raw sequence and $99.4 \%$ exome regions were covered at least four times. A summary of the identified variants was shown in Table I, including 451,409 and 19,995 filtered SNVs and small indels, respectively.

Concordance of SNVs identified by WGS and WES. The genotype concordance between WGS and WES was high. Of $438,261 \mathrm{SNV}$ s called in both sequencing data, genotypes in 395,824 SNVs $(90.32 \%)$ were consistent. Nearly all of inconsistent genotypes $(42,327$ out of $42,437,99.7 \%)$ resulted from a low sequence depth in either WGS or WES data, leading to alternative-allele homozygous calls. The remaining sites of inconsistent genotypes may be introduced by sequencing errors. Therefore, we estimated the sequence error rate to be $0.024 \%$ (110/42437). Of $36 \mathrm{SNVs}$ listed in Table II, genotypes of $31 \mathrm{SNVs}$ were consistently identified in both sequencing, and the remaining 5 were only identified in WGS. We also estimated the false discovery rate of novel SNV identification by randomly selecting 53 novel SNVs identified in both WGS and WES for validation by Sanger sequencing. We obtained 47 and 49 PCR products for pre-AML and AML-DNA, respectively. Of 41 and 39 SNVs with successful sequencing, only one SNV for each were found to be false positive (false discovery rate, 2.4 and $2.6 \%$, respectively). 
Table II. Identified rare missense mutations in genes implicated in hereditary cancer predisposition syndromes and mismatch repair genes.

\begin{tabular}{|c|c|c|c|c|c|}
\hline Chr_pos ${ }^{\mathrm{a}}$ & Rs ID & AA change ${ }^{b}$ & PolyPhen ${ }^{c}$ & Asian HapMapFreq ${ }^{\mathrm{d}}$ & Gene \\
\hline \multicolumn{6}{|c|}{ Hereditary cancer predisposition syndromes } \\
\hline $7: 6026942$ & rs1805323 & $\mathrm{T}->\mathrm{K}$ & 0 & NA & $P M S 2$ \\
\hline $7: 6026988$ & rs1805321 & $\mathrm{P}->\mathrm{S}$ & 0.018 & 0 & $P M S 2$ \\
\hline $7: 142460335$ & rs201550522 & $\mathrm{K}->\mathrm{E}$ & 0 & NA & PRSS1 \\
\hline $7: 142460369$ & rs201719096 & $\mathrm{S}->\mathrm{N}$ & 0 & NA & PRSS1 \\
\hline $7: 142460744$ & rs150930992 & $\mathrm{C}->\mathrm{S}$ & 0.185 & NA & PRSS1 \\
\hline $7: 142460752$ & rs140793689 & $\mathrm{Q}->\mathrm{E}$ & 0 & NA & PRSS1 \\
\hline $7: 142460764$ & rs200902389 & $\mathrm{V}->\mathrm{I}$ & 0 & NA & PRSS1 \\
\hline $8: 145737816$ & rs4251691 & $\mathrm{R}->\mathrm{Q}$ & 0.035 & NA & $R E C Q L 4$ \\
\hline $8: 145741702$ & rs4244612 & $E->D$ & 0.002 & NA & RECQLA \\
\hline $8: 145742514$ & rs2721190 & $\mathrm{S}->\mathrm{P}$ & Unknown & 0 & RECQLA \\
\hline $9: 135772897$ & - & $\mathrm{I}->\mathrm{T}$ & 0.77 & NA & TSC1 \\
\hline $10: 88635779$ & rs11528010 & $\mathrm{P}->\mathrm{T}$ & 0 & NA & BMPR1A \\
\hline $10: 14976727$ & rs35441642 & $\mathrm{P}->\mathrm{R}$ & 1 & NA & DCLREIC \\
\hline $11: 111963860$ & rs592626 & Q->R & Unknown & NA & $S D H D$ \\
\hline $13: 32929387$ & rs169547 & $\mathrm{V}->\mathrm{A}$ & Unknown & 0.7 & $B R C A 2$ \\
\hline $15: 91354521$ & rs7167216 & $\mathrm{V}->\mathrm{I}$ & 0.01 & 3.3 & $B L M$ \\
\hline $16: 68856080$ & rs2276331 & $\mathrm{L}->\mathrm{V}$ & 1 & 1.7 & $C D H 1$ \\
\hline $16: 89813029$ & - & $\mathrm{C}->\mathrm{S}$ & 1 & NA & $F A N C A$ \\
\hline $16: 89836323$ & rs7195066 & G->D & 0 & 1.2 & $F A N C A$ \\
\hline $16: 89866043$ & rs7190823 & $\mathrm{T}->\mathrm{A}$ & 0 & 1.9 & $F A N C A$ \\
\hline $17: 7577120$ & rs28934576 & $\mathrm{R}->\mathrm{H}$ & 1 & NA & TP53 \\
\hline $19: 1223125$ & rs59912467 & $\mathrm{F}->\mathrm{L}$ & 0.062 & NA & STK11 \\
\hline \multicolumn{6}{|c|}{ Mismatch repair genes } \\
\hline 2:100055158 & rs3087399 & $\mathrm{N}->\mathrm{S}$ & 0.069 & 3.6 & $R E V 1$ \\
\hline $3: 8977648$ & - & $\mathrm{S}->\mathrm{P}$ & 0.937 & NA & $R A D 18$ \\
\hline $3: 121263720$ & rs702017 & $\mathrm{R}->\mathrm{I}$ & 0 & 0 & $P O L Q$ \\
\hline $5: 68531253$ & rs2972388 & $\mathrm{T}->\mathrm{I}$ & Unknown & NA & $C D K 7$ \\
\hline $5: 68695940$ & rs1045051 & $\mathrm{L}->\mathrm{R}$ & 1 & 0 & $R A D 17$ \\
\hline $5: 80149981$ & rs184967 & Q->R & 0 & 0 & MSH3 \\
\hline $7: 6026942$ & rs1805323 & $\mathrm{T}->\mathrm{K}$ & 0 & NA & $P M S 2$ \\
\hline $7: 6026988$ & rs1805321 & $\mathrm{P}->\mathrm{S}$ & 0.018 & 0 & $P M S 2$ \\
\hline $8: 95403868$ & rs114216685 & $\mathrm{N}->\mathrm{S}$ & 1 & NA & $R A D 54 B$ \\
\hline $10: 99240758$ & rs2275586 & $A->G$ & 0 & 2.8 & MMS19 \\
\hline $11: 125525195$ & rs506504 & $\mathrm{I}->\mathrm{V}$ & 0 & 0 & CHEK1 \\
\hline $12: 104380734$ & rs2888805 & $\mathrm{V}->\mathrm{M}$ & 0.833 & NA & $T D G$ \\
\hline $14: 75513883$ & rs175081 & $\mathrm{N}->\mathrm{D}$ & 0.001 & 0.4 & MLH3 \\
\hline 19:7692266 & - & $\mathrm{T}->\mathrm{P}$ & 0.961 & NA & $X A B 2$ \\
\hline $19: 44047826$ & rs2682557 & $\mathrm{N}->\mathrm{Y}$ & 0 & NA & $X R C C 1$ \\
\hline $19: 50370425$ & rs201218221 & $E->Q$ & 0.004 & NA & $P N K P$ \\
\hline
\end{tabular}

${ }^{a}$ Chromosome and position in $\mathrm{Hg} 19$; ${ }^{b}$ amino acid change; ${ }^{c}$ a larger score of PolyPhen indicated a possible or probably damaging effect; ${ }^{\mathrm{d}}$ frequency in HapMap Asian population (\%). PMS2, postmeiotic segregation increased 2; PRSS1, protease, serine, 1 (trypsin 1); RECQL4, RecQ protein-like 4; TSC1, tuberous sclerosis 1; BMPRIA, bone morphogenetic protein receptor, type IA; DCLRE1C, DNA cross-link repair $1 \mathrm{C}$; $S D H D$, succinate dehydrogenase complex, subunit D, integral membrane protein; BRCA2, breast cancer 2, early onset; $B L M$, Bloom syndrome, RecQ helicase-like; $C D H 1$, cadherin 1, type 1, E-cadherin (epithelial); FANCA, Fanconi anemia, complementation group A; TP53, tumor protein p53; and STK11, serine/threonine kinase 11; CDK7, cyclin-dependent kinase 7; CHEK1, checkpoint kinase 1; MLH3, mutL homolog 3; MMS19, MMS19 nucleotide excision repair homolog (S. cerevisiae); MSH3, mutS homolog 3; PMS2, PMS2 postmeiotic segregation increased 2; PNKP, polynucleotide kinase 3'-phosphatase; $P O L Q$, polymerase (DNA directed), theta; RAD17, RAD17 homolog (S.pombe); $R A D 18, \mathrm{RAD} 18 \mathrm{E} 3$ ubiquitin protein ligase; $R A D 54 B, \mathrm{RAD} 54$ homolog B (S. cerevisiae); REV1, REV1, polymerase (DNA directed); TDG, thymine-DNA glycosylase; $X A B 2$, XPA binding protein 2; XRCC1, X-ray repair complementing defective repair in Chinese hamster cells 1. 


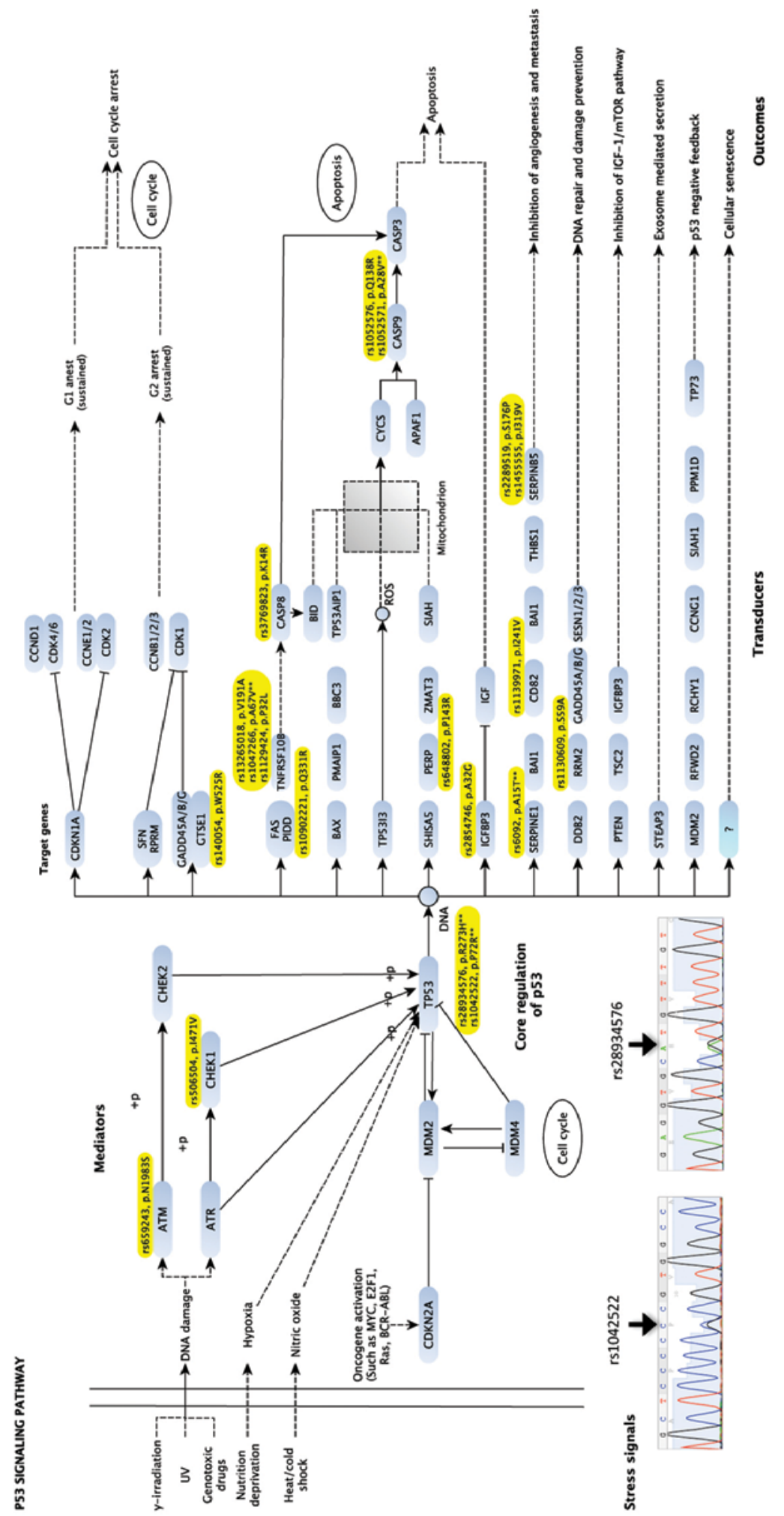

Figure 3. Missense mutations identified in the p53-signaling pathway. The image was taken and abridged from the KEGG database. Missense mutation marked with amino acid change is highlighted. The asterisks $(* *)$ indicate probably or possibly damaging mutations based on PolyPhen predictions. Inserted chromatograms showed two missense mutations (rs28934576 and rs1042522) in TP53 by Sanger sequencing. 


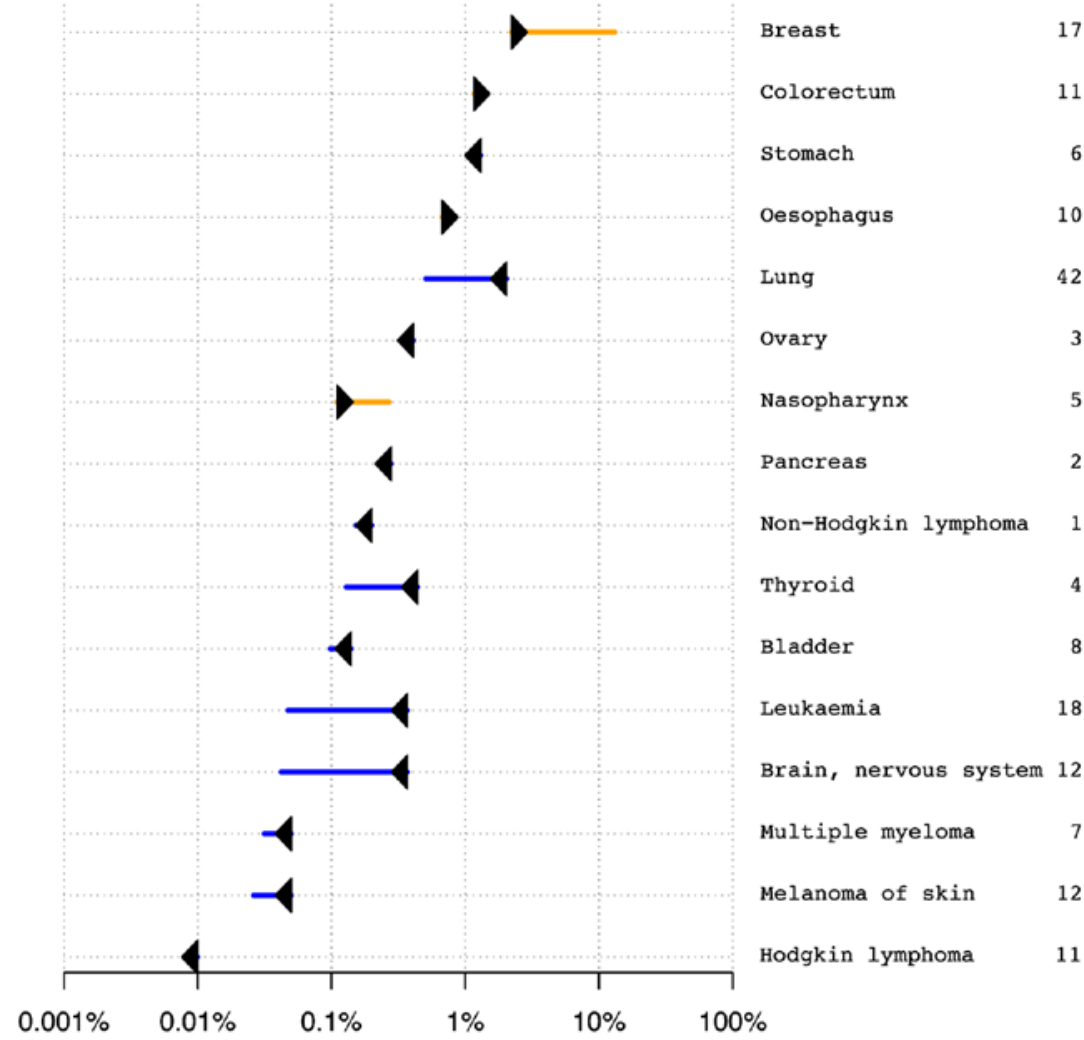

Figure 4. Overall Risk-O-Gram for diverse cancer types. Cancers were listed on the right together with the number of independent LD groups that the prediction was based upon. The $\mathrm{x}$-axis represented the probability of developing cancer shown on the $\log _{10}$ scale. The triangles showed the pre-test probabilities whereas the lines show the post-test probabilities. The orange and blue lines correspond to increased and decreased risk, respectively.

WES-based cancer risk evaluation: Risk-O-Gram analysis. It is unknown whether the phenotypes of multiple primary malignant neoplasms in the studied patient were caused by an unusual accumulation of common cancer risk variants. VARIMED-based analysis of the genetic risk of cancer did not reveal increased risk for multiple cancers (Fig. 4). Risk-OGram plots for four cancers (breast, gastric, neuroblastoma and thyroid) diagnosed in the patient were available upon request. Our results suggested that the patient yielded an average overall risk of cancer, with an increased risk of some cancers and decreased risk of others. For example, a significantly increased risk of breast cancer was estimated to be over six times greater than expected. Only $2 \%$ of control samples have higher predicted risk of breast cancer. The patient developed right breast cystosarcoma phyllodes at 28 years and left breast infiltrating ductal carcinoma at 39 years, which confirmed our predictions. An increased risk of developing nasopharyngeal cancer was predicted, which, however, was not diagnosed in the patient. Regarding other diagnosed and undiagnosed cancers, the patient showed either average risk, not deviating from the median of the control samples (e.g. neuroblastoma and lung cancer), or significantly decreased risk (e.g. thyroid cancer and melanoma) (data not shown). The remaining cancers diagnosed in the patient did not have enough significant associations in VARIMED.

In addition, in order to assess the patient's risk with respect to the control population, we performed the risk calculation for all Chinese samples of the 1000 Genomes data (15). The fractions of control samples (CHB and CHS populations from the
1000 Genomes Project) have lower, equal or higher predicted genetic risk than the cancer risk of the studied patient (data not shown).

Somatic mutations implicated in leukemic transformation. We compared the sequencing data of AML-DNA with preAML-DNA, and identified at least $60 \mathrm{mis}-/$ non-sense somatic mutations. Of note, 12 missense mutations in 11 genes have been reported in COSMIC database (29), including MLL3, that may contribute to development of $\mathrm{AML}^{-\mathrm{M}_{2}}$. It has been suggested that $M L L 3$ is a haploinsufficient 7q tumor suppressor in AML (30), and a germline mutation in MLL3 was identified in a pedigree of colorectal cancer and AML (31).

\section{Discussion}

We presented a rare case of Li-Fraumeni syndrome (LFS), who had developed at least nine primary malignant neoplasms over a period of 38 years. The characteristics of this patient, i.e., presenting with cancers at an early age (7 years), with multiple primary cancers, and with a family history, suggested an increased inherited cancer susceptibility risk. The identification of the genetic basis for cancer susceptibility has important clinical implications for the early diagnosis and prevention (e.g., change of lifestyle) of associated neoplasms. A recent study has shown that regular screening in LFS families led to a survival benefit (32). The advent of next-generation sequencing, a comprehensive, unbiased approach, made it possible to identify mutations in cancer susceptibility genes. 
In the present study, we identified rare mutations leading to multiple malignant neoplasms, retrospectively estimated cancer risk, and explored the underlying mechanism of her relatively longer life expectancy.

We found mutations in cancer susceptibility genes of hereditary breast cancer syndromes (e.g., LFS), including TP53 and BRCA2. The mutations in TP53 may impair or reduce the function of p53 due to dominant negative activity (33). Mutations in TP53 correlated with 'complex' karyotypes in AML (34) as shown in the bone marrow of the studied patient. The number of mutated genes in the p53 signaling pathway was significantly greater than expected, which suggested that, the p53 stress response pathway harbors functional inherited variants acting together to affect p53 signaling in cells (35). The patient contained rare mutations in five genes (CDH1, BMPRIA, STK11, PRSS1 and $P M S 2)$, which were implicated in hereditary gastrointestinal malignancies. A mutation in $\mathrm{CDH}$ l was found to be associated with an increased risk developing gastric cancer, e.g., hereditary diffuse gastric cancer (36).

It is possible that an accumulation of common germline variants associated with cancer may determine inherited predisposition to cancer. To test the hypothesis, we estimated cancer risk by integrating information from multiple alleles associated with cancer risk. We found that the phenotype of the studied patient, characterized by the development of multiple cancers, was unlikely to be caused by the unusual accumulation of known cancer-associated common variants (Fig. 4), although the patient did have an increased risk of breast cancer when compared with the control populations in the 1000 Genomes Project. However, the post-probabilities did not take into account the exactly matching background population; for example, the predicted genetic risk of lung cancer was decreased not only in the studied patient but also in the studied background population. It is unknown whether rare mutations with high penetrance and common low penetrant variants have synergistic effects in the development of multiple cancers in the patient.

Life expectancy is substantially reduced in LFS, which is likely to be below 40 years of age on average (37). Given the development of nine malignant tumors in the patient, the survival (45 years) was better than expected. One reason was that the patient received excellent health care at the PUMCH. Second, we could consider an alternative hypothesis that the patient's genome is depleted of cancer risk alleles and enriched for cancer protective alleles. Our disease-based analysis of known variants seemed to partially support this hypothesis in that the total number of risk alleles in the patient was less than the average in control samples. However, VARIMED does not really provide evidence that the patient was protected from dying from cancer (SNPs in VARIMED are the risk SNPs for developing a cancer but not for survival).

Other possible explanations for the occurrence of nine primary metachronous malignant neoplasms in the patient included: radiation-induced cancers have been suggested to be more common in patients with LFS $(38,39)$; and therapyrelated AML is a rare but well-described complication of chemotherapy (40). In the setting of DNA repair defects due to germline TP53 mutation, the effect of therapeutic ionizing radiation is a major concern. We could not exclude the possi- bility that AML in this patient is secondary after etoposide therapy for bronchioalveolar cell carcinoma (41).

It has been suggested that whether a TP53 germline mutation carrier will develop cancer and where the cancer(s) will develop depends upon where the second mutation occurs in which cell type. It is unclear whether there was a second TP53 mutation in the patient. Typically, a tumor contains two to eight of 'driver gene' mutations (42), thus, it is unknown whether there were driver somatic mutations or massive chromosome rearrangements underlying the development of different tumors. Discovering mutation driver genes for different tumors in the studied patient remains challenging. Rausch et al (43) uncovered massive, complex chromosome rearrangements (termed 'chromothripsis') in a Sonic-Hedgehog medulloblastoma brain tumor from an LFS patient. The association between TP53 mutation and chromothripsis in Sonic-Hedgehog medulloblastoma was revealed in additional patients (43). More work is needed to characterize structural variants, especially for chromothripsis, in different tumors.

In conclusion, we identified multiple rare mutations in genes implicated in hereditary cancer predisposition syndromes, which may lead to multiple malignant neoplasms in the studied patient. Of note, we found that the number of mutated genes in p53 signaling pathway was significantly greater than expected. However, the phenotype of multiple primary neoplasms in this patient was unlikely to be caused by an unusual accumulation of common cancer risk variants. Further studies are needed to characterize mutation patterns, including somatic mutations and structural variants in different tumors developed in this patient.

\section{Acknowledgements}

We thank Di Ma, Xiao Chen, Wenxun Huang, Xia Tang, Wenwei Yin and Shiwen Tong for technical support. The study is supported by the Recruitment Program of Global Youth Experts in China (KD) and a start-up fund from the Second Affiliated Hospital of Chongqing Medical University (KD). Conflict of interest: A. Patwardhan and R. Chen are employees of Personalis, Inc., the company licenced to develop the VARIMED database.

\section{References}

1. Li FP and Fraumeni JF Jr: Soft-tissue sarcomas, breast cancer, and other neoplasms. A familial syndrome? Ann Intern Med 71: 747-752, 1969.

2. Hisada M, Garber JE, Fung CY, Fraumeni JF Jr and Li FP: Multiple primary cancers in families with Li-Fraumeni syndrome. J Natl Cancer Inst 90: 606-611, 1998.

3. Olivier M, Hollstein M and Hainaut P: TP53 mutations in human cancers: Origins, consequences, and clinical use. Cold Spring Harb Perspect Biol 2: a001008, 2010.

4. Bell DW, Varley JM, Szydlo TE, Kang DH, Wahrer DC, Shannon KE, Lubratovich M, Verselis SJ, Isselbacher KJ, Fraumeni JF, et al: Heterozygous germ line hCHK2 mutations in Li-Fraumeni syndrome. Science 286: 2528-2531, 1999.

5. Bachinski LL, Olufemi S-E, Zhou X, Wu CC, Yip L, Shete S, Lozano G, Amos CI, Strong LC and Krahe R: Genetic mapping of a third Li-Fraumeni syndrome predisposition locus to human chromosome 1q23. Cancer Res 65: 427-431, 2005.

6. Chen K, Wallis JW, McLellan MD, Larson DE, Kalicki JM, Pohl CS, McGrath SD, Wendl MC, Zhang Q, Locke DP, et al: BreakDancer: An algorithm for high-resolution mapping of genomic structural variation. Nat Methods 6: 677-681, 2009. 
7. Boeva V, Popova T, Bleakley K, Chiche P, Cappo J Schleiermacher G, Janoueix-Lerosey I, Delattre $\mathrm{O}$ and Barillot E: Control-FREEC: A tool for assessing copy number and allelic content using next-generation sequencing data. Bioinformatics 28: 423-425, 2012.

8. Cibulskis K, Lawrence MS, Carter SL, Sivachenko A, Jaffe D, Sougnez C, Gabriel S, Meyerson M, Lander ES and Getz G: Sensitive detection of somatic point mutations in impure and heterogeneous cancer samples. Nat Biotechnol 31: 213-219, 2013.

9. Gilissen C, Hoischen A, Brunner HG and Veltman JA: Disease gene identification strategies for exome sequencing. Eur J Hum Genet 20: 490-497, 2012.

10. Garber JE and Offit K: Hereditary cancer predisposition syndromes. J Clin Oncol 23: 276-292, 2005.

11. Wood RD, Mitchell M, Sgouros J and Lindahl T: Human DNA repair genes. Science 291: 1284-1289, 2001.

12. Ramensky V, Bork P and Sunyaev S: Human non-synonymous SNPs: Server and survey. Nucleic Acids Res 30: 3894-3900, 2002.

13. Chen R, Davydov EV, Sirota M and Butte AJ: Non-synonymous and synonymous coding SNPs show similar likelihood and effect size of human disease association. PLoS One 5: e13574, 2010.

14. Ashley EA, Butte AJ, Wheeler MT, Chen R, Klein TE, Dewey FE, Dudley JT, Ormond KE, Pavlovic A, Morgan AA, et al: Clinical assessment incorporating a personal genome. Lancet 375: $1525-1535,2010$

15. 1000 Genomes Project Consortium, Abecasis GR, Auton A, Brooks LD, DePristo MA, Durbin RM, Handsaker RE, Kang HM, Marth GT and McVean GA: An integrated map of genetic variation from 1,092 human genomes. Nature 491: 56-65, 2012.

16. Mwenifumbo JC and Marra MA: Cancer genome-sequencing study design. Nat Rev Genet 14: 321-332, 2013.

17. Malkin D, Jolly KW, Barbier N, Look AT, Friend SH, Gebhardt MC, Andersen TI, Børresen AL, Li FP, Garber J, et al: Germline mutations of the p53 tumor-suppressor gene in children and young adults with second malignant neoplasms. $\mathrm{N}$ Engl J Med 326: 1309-1315, 1992.

18. Sugano K, Taniguchi T, Saeki M, Tsunematsu Y, Tomaru U and Shimoda T: Germline p53 mutation in a case of Li-Fraumeni syndrome presenting gastric cancer. Jpn J Clin Oncol 29: 513-516, 1999.

19. Healey CS, Dunning AM, Teare MD, Chase D, Parker L, Burn J, Chang-Claude J, Mannermaa A, Kataja V, Huntsman DG, et al: A common variant in BRCA2 is associated with both breast cancer risk and prenatal viability. Nat Genet 26: 362-364, 2000.

20. Masciari S, Larsson N, Senz J, Boyd N, Kaurah P, Kandel MJ, Harris LN, Pinheiro HC, Troussard A, Miron P, et al: Germline E-cadherin mutations in familial lobular breast cancer. J Med Genet 44: 726-731, 2007.

21. Kaurah P, MacMillan A, Boyd N, Senz J, De Luca A, Chun N, Suriano G, Zaor S, Van Manen L, Gilpin C, et al: Founder and recurrent $\mathrm{CDH} 1$ mutations in families with hereditary diffuse gastric cancer. JAMA 297: 2360-2372, 2007.

22. Mohaghegh P and Hickson ID: DNA helicase deficiencies associated with cancer predisposition and premature ageing disorders. Hum Mol Genet 10: 741-746, 2001.

23. Kitao S, Shimamoto A, Goto M, Miller RW, Smithson WA Lindor NM and Furuichi Y: Mutations in RECQL4 cause a subset of cases of Rothmund-Thomson syndrome. Nat Genet 22 : 82-84, 1999.

24. Ellis NA, Groden J, Ye TZ, Straughen J, Lennon DJ, Ciocci S, Proytcheva $\mathrm{M}$ and German J: The Bloom's syndrome gene product is homologous to RecQ helicases. Cell 83: 655-666, 1995.

25. Thompson ER, Doyle MA, Ryland GL, Rowley SM, Choong DY, Tothill RW, Thorne H, Barnes DR, Li J, Ellul J, et al; kConFab: Exome sequencing identifies rare deleterious mutations in DNA repair genes FANCC and BLM as potential breast cancer susceptibility alleles. PLoS Genet 8: e1002894, 2012.
26. Kunkel TA and Erie DA: DNA mismatch repair. Annu Rey Biochem 74: 681-710, 2005.

27. Nakagawa H,Lockman JC, Frankel WL, Hampel H, Steenblock K, Burgart LJ, Thibodeau SN and de la Chapelle A: Mismatch repair gene PMS2: Disease-causing germline mutations are frequent in patients whose tumors stain negative for PMS2 protein, but paralogous genes obscure mutation detection and interpretation. Cancer Res 64: 4721-4727, 2004.

28. Hitchins MP, Wong JJL, Suthers G, Suter CM, Martin DIK, Hawkins NJ and Ward RL: Inheritance of a cancer-associated MLH1 germ-line epimutation. N Engl J Med 356: 697-705, 2007.

29. Forbes SA, Bindal N, Bamford S, Cole C, Kok CY, Beare D, Jia M, Shepherd R, Leung K, Menzies A, et al: COSMIC: Mining complete cancer genomes in the Catalogue of Somatic Mutations in Cancer. Nucleic Acids Res 39 (Database): D945D950, 2011.

30. Chen C, Liu Y, Rappaport AR, Kitzing T, Schultz N, Zhao Z, Shroff AS, Dickins RA, Vakoc CR, Bradner JE, et al: MLL3 is a haploinsufficient $7 \mathrm{q}$ tumor suppressor in acute myeloid leukemia. Cancer Cell 25: 652-665, 2014.

31. Li WD, Li QR, Xu SN, Wei FJ, Ye ZJ, Cheng JK and Chen JP: Exome sequencing identifies an MLL3 gene germ line mutation in a pedigree of colorectal cancer and acute myeloid leukemia. Blood 121: 1478-1479, 2013.

32. Villani A, Tabori U, Schiffman J, Shlien A, Beyene J, Druker H, Novokmet A, Finlay J and Malkin D: Biochemical and imaging surveillance in germline TP53 mutation carriers with Li-Fraumeni syndrome: A prospective observational study. Lancet Oncol 12: 559-567, 2011.

33. Vogelstein B, Lane D and Levine AJ: Surfing the p53 network. Nature 408: 307-310, 2000.

34. Haferlach C, Dicker F, Herholz H, Schnittger S, Kern W and Haferlach T: Mutations of the TP53 gene in acute myeloid leukemia are strongly associated with a complex aberrant karyotype. Leukemia 22: 1539-1541, 2008.

35. Grochola LF, Zeron-Medina J, Mériaux S and Bond GL: Singlenucleotide polymorphisms in the p53 signaling pathway. Cold Spring Harb Perspect Biol 2: a001032, 2010.

36. Fitzgerald RC, Hardwick R, Huntsman D, Carneiro F, Guilford P, Blair V, Chung DC, Norton J, Ragunath K, Van Krieken JH, et al; International Gastric Cancer Linkage Consortium: Hereditary diffuse gastric cancer: Updated consensus guidelines for clinical management and directions for future research. J Med Genet 47: 436-444, 2010.

37. Evans DGR and Ingham SL: Reduced life expectancy seen in hereditary diseases which predispose to early-onset tumors. Appl Clin Genet 6: 53-61, 2013

38. Limacher JM, Frebourg T, Natarajan-Ame S and Bergerat JP: Two metachronous tumors in the radiotherapy fields of a patient with Li-Fraumeni syndrome. Int J Cancer 96: 238-242, 2001.

39. Heymann S, Delaloge S, Rahal A, Caron O, Frebourg T, Barreau L, Pachet C, Mathieu MC, Marsiglia H and Bourgier C: Radio-induced malignancies after breast cancer postoperative radiotherapy in patients with Li-Fraumeni syndrome. Radiat Oncol 5: 104, 2010.

40. Godley LA and Larson RA: Therapy-related myeloid leukemia. Semin Oncol 35: 418-429, 2008.

41. Smith MA, Rubinstein L, Anderson JR, Arthur D, Catalano PJ, Freidlin B, Heyn R, Khayat A, Krailo M,Land VJ, et al: Secondary leukemia or myelodysplastic syndrome after treatment with epipodophyllotoxins. J Clin Oncol 17: 569-577, 1999.

42. Vogelstein B, Papadopoulos N, Velculescu VE, Zhou S, Diaz LA Jr and Kinzler KW: Cancer genome landscapes. Science 339: 1546-1558, 2013

43. Rausch T, Jones DTW, Zapatka M, Stütz AM, Zichner T, Weischenfeldt J, Jäger N, Remke M, Shih D, Northcott PA, et al: Genome sequencing of pediatric medulloblastoma links catastrophic DNA rearrangements with TP53 mutations. Cell 148: 59-71, 2012. 